\section{Clinical guide to periodontology: part 3. Multidisciplinary integrated treatment}

\author{
R. M Palmer, ${ }^{* 1}$ M. Ide ${ }^{2}$ and P. D. Floyd ${ }^{3}$
}
IN BRIEF
- Stresses drifted anterior teeth may be a sign of severe periodontitis. Management may include periodontal treatment, splinting and supportive care.
- Informs crown lengthening surgery is one of the commonest pre-restorative periodontal procedures.
- Highlights replacement of missing teeth using dental implants in periodontitis patients requires appropriate treatment of remaining teeth.

The establishment of periodontal health should be a primary aim in all treatment plans. The methods by which this can be achieved have been dealt with in previous chapters, but there are a number of situations where integration of these treatment methods with other dental disciplines needs to be clarified. To simplify matters this chapter will consider periodontal implications in three main areas: treatment of drifted anterior teeth, pre-restorative procedures and replacement of missing teeth.

\section{DRIFTED ANTERIOR TEETH}

Drifting or spacing of the maxillary anterior teeth is a frequent complaint of patients with periodontitis and demands careful diagnostic evaluation before choosing the treatment options. This is because the cause of the problem is usually multifactorial. The evaluation can therefore be conveniently divided into three sections:

- Periodontal

- Orthodontic

- Restorative/occlusal.

\section{EVALUATION OF AETIOLOGY}

\section{Periodontal}

In situations where occlusal or soft tissue forces are persistent and likely to produce movement of teeth, the required magnitude

\section{CLINICAL GUIDE TO PERIODONTOLOGY*}

1. Pathology of periodontal disease

2. Reconstructive periodontal treatment 3. Multidisciplinary integrated treatment "This series represents chapters 2, 9 and 10 from the BDJ Book A clinical guide to periodontology, 3rd ed, edited by Richard Palmer, Mark Ide and Peter Floyd. All other chapters are published in the complete clinical guide available from the BDJ Books online shop.
published in the complet
${ }^{1}$ Professor of Implant Dentistry and Periodontology, King's College London Dental Institute, London; ${ }^{2} \mathrm{Head}$ of Periodontology, King's College London Dental Institute London, ${ }^{3}$ Specialist in Periodontics, Principal, Periodontics UK, Wimpole Street, London *Correspondence to Richard Palmer Email:rich.m.palmer@gmail.com

DOI: 10.1038/sj.bdj.2014.400

${ }^{\circledR}$ British Dental Journal 2014; 216: 567-573 of force will be inversely proportional to the amount of periodontal support. Chronic periodontitis is the most common cause of destruction of the periodontal support. Inflammation destroys the integrity of local interdental collagen bundles and consequently alters the equilibrium stabilising the buccopalatal position of the tooth. The pattern of attachment loss on an individual tooth is also pertinent. In many cases the deepest pocketing and the most severe bone loss is on the palatal aspect of a labially migrated incisor. This has led to the (unproven) proposal that the forces generated within the inflammatory lesion are responsible for the tooth movement. Recurrent abscesses in this situation may lead to rapid destruction and drifting. The amount of periodontal support will also depend upon factors such as:

- Root length

- Root shape (for example, very tapering root forms)

- Root resorption (for example, post orthodontic)

- Endodontic lesions destroying the apical periodontium.

These factors can be accurately assessed by good-quality intraoral radiographs, which will also confirm the degree of bone loss estimated from the clinical probing examination.

All these factors contribute to the mobility of the teeth, which is further increased by inflammation and occlusal forces. The term 'jiggling forces' applies to the situation where an occlusal force moves the tooth in one direction and soft tissue forces move it back to the original position. Mobility, which can be seen or felt (by the clinician's fingers
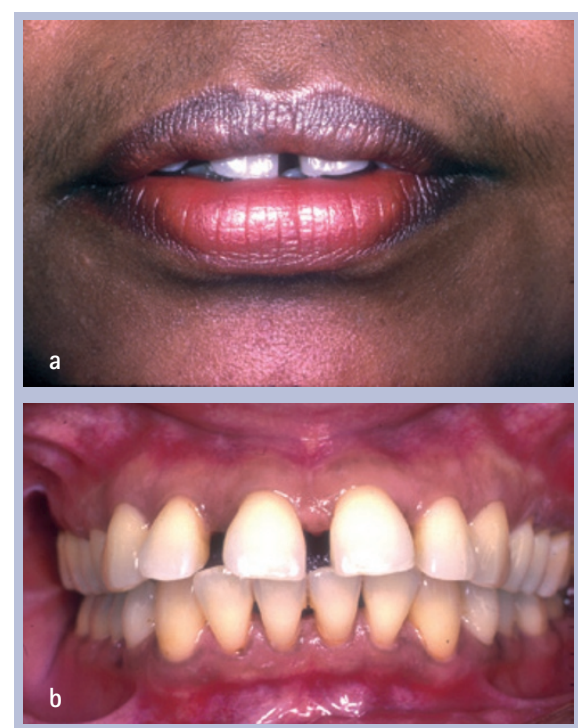

Figs $1 \mathrm{a}$ and $\mathrm{b}$ Clinical photographs of drifted upper central incisors not within the control of the lower lip

placed gently on the labial surface of the incisors) when the patient gently occludes is known as fremitus and is an indication of 'jiggling'.

\section{Orthodontic}

It is important to establish whether the patient has previously had orthodontic correction of a Class II division 1 incisor relationship that is no longer stable; that is, relapse of a previously treated malocclusion. Most individuals with drifting incisors (Fig. 1) have a pre-existing tendency to this incisor relationship and incompetent lip morphology. In many cases it is only one or two of the incisors that escape the control of the lower lip, and any tendency to lip bite will accentuate the situation. 
Other contributory parafunctional habits, such as patients who persistently bite on a foreign object, are more rarely encountered. At completion of the treatment plan the incisors will need to be placed in a position of stability within the soft tissue pattern and adequate space is essential to achieve this. Permanent retention is required if the orthodontic result is prone to relapse. It is prudent to seek the advice of an orthodontic specialist, particularly if repositioning is being considered.

\section{Restorative/occlusal}

There are a number of potential restorative factors which may be associated with this problem:

- Loss of posterior support causing the patient to function on the anterior teeth and often associated with forward posturing of the mandible

- Recent provision of anterior crowns that have altered the incisal guidance

- Occlusal interferences which may precipitate parafunctional activities.

The static and functional relationships of the teeth should be examined. It is particularly important to assess the incisal guidance and whether there is a marked horizontal discrepancy between the retruded contact position and the intercuspal position. The protrusive and lateral excursion contacts should be evaluated for fremitus and interferences (especially non-working).

\section{TREATMENT OPTIONS}

The treatment options should be more apparent after considering the aetiological factors in addition to the prognostic factors of the individual teeth and dentition as a whole. There are basically three options:

- Accept position of teeth

- Orthodontic repositioning

- Extraction and replacement.

\section{Accept position of teeth}

The position of the drifted teeth may be acceptable to the patient, particularly if the situation is unlikely to deteriorate further. Further drifting may be reduced with successful treatment of the periodontitis and attention to occlusal factors:

- Selective grinding to eliminate occlusal interferences (particularly those associated with forward positioning of the mandible) and reduce fremitus

- Replacement of unsatisfactory restorations

- Provision of a posterior occlusion if possible and acceptable to the patient

- Provision of occlusal guard to dissipate parafunctional forces.
In some cases improvement of the tooth position can occur with this treatment. If the factors operating on the teeth are large then it is impossible to give the patient firm reassurance that the situation is stable.

Many patients faced with the other options are prepared to accept the situation if the aesthetics are not too compromised.

\section{Orthodontic repositioning}

This is the most demanding of the treatment options and should not be entered into lightly. There are basic guiding rules:

- The periodontal condition must be treated initially

- Periodontal inflammation must be controlled during active tooth movement

- The orthodontic forces have to be carefully controlled

- In most cases the orthodontic result will not be stable without permanent splinting.

Orthodontic tooth movement in untreated periodontitis is likely to result in further loss of attachment and in severe cases abscess formation and rapid destruction. The minimum periodontal treatment is thorough root surface debridement and establishment of a high standard of supragingival plaque control by the patient. The provision of an orthodontic appliance may compromise plaque control and extra effort is required by the patient. If the orthodontic treatment is likely to be prolonged, then further and repeated subgingival instrumentation and maintenance treatment will be required during this phase.

In cases where periodontal surgery is required this can often be delayed until after the orthodontic work has been completed. This has the advantage of re-establishing a dentogingival junction around the teeth in their corrected position, and subsequent maturation of the supracrestal collagen fibre arrangement may enhance stability.

While some simple cases can be managed with removable appliances, many will require fixed appliances and the services of a specialist orthodontist. In the latter case it is helpful if the orthodontist has had experience of moving teeth with compromised support. It is particularly important to avoid overloading and complications such as root resorption.

Most orthodontists will give no guarantee of stability of the end result in anything but the simplest of cases, and usually recommend permanent splinting. Splinting systems that rely on resin-bonded restorations may give adequate service in some instances. They range from simple wire and composite splints to Rochette and Maryland-type retainers. These restorations are not as predictable as one would wish for such complex integrated treatment plans. Rochette frameworks have
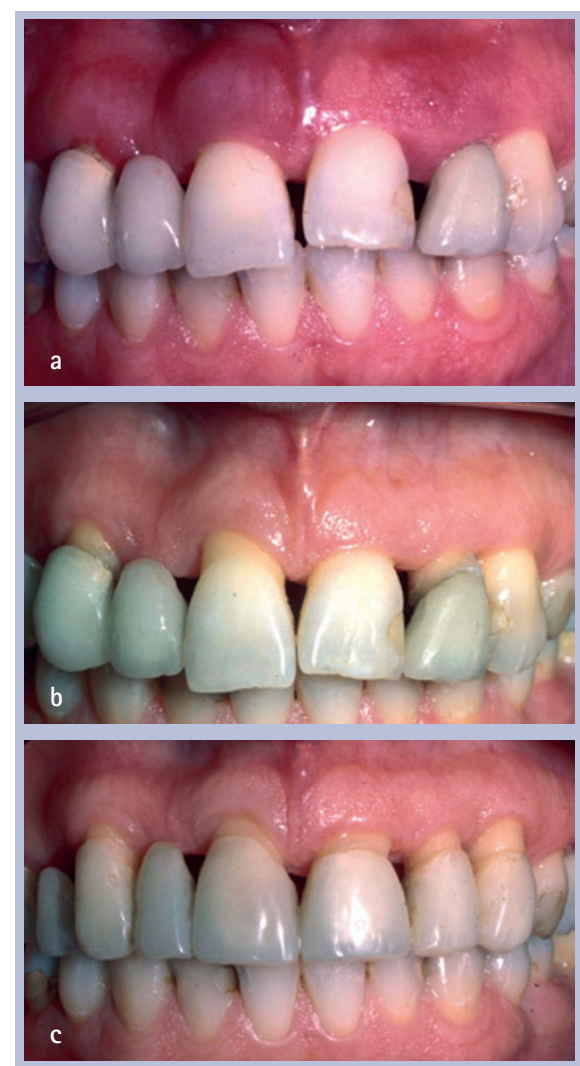

Fig. 2 a) Drifted upper incisors with moderate periodontitis. b) Following non-surgical periodontal treatment and orthodontic repositioning. c) Following periodontal surgery to eliminate residual pockets and splinting to maintain the result

the advantage of being more easily removed if recementation is required.

Successful, predictable long-term retention may need an extensive restoration. In some cases this is only achievable with conventional full-coverage restorations. Under these circumstances due consideration needs to be given to extracting all or some of the drifted teeth in the first place. This may eliminate the need for orthodontics. Treatment of a patient involving orthodontics, periodontics and splinting is shown in Figure 2.

\section{PRE-RESTORATIVE PERIODONTAL PROCESDURES}

In addition to the establishment of periodontal health, periodontal surgical techniques can be usefully applied in the following areas:

- Crown lengthening

- Ridge augmentation

- Gingival augmentation.

Crown lengthening and ridge augmentation are covered in this chapter and gingival augmentation is dealt with in part two.

\section{Crown lengthening}

The periodontal surgical techniques described in Chapter 7 of the $B D J$ clinical guide associated with this series can be usefully 

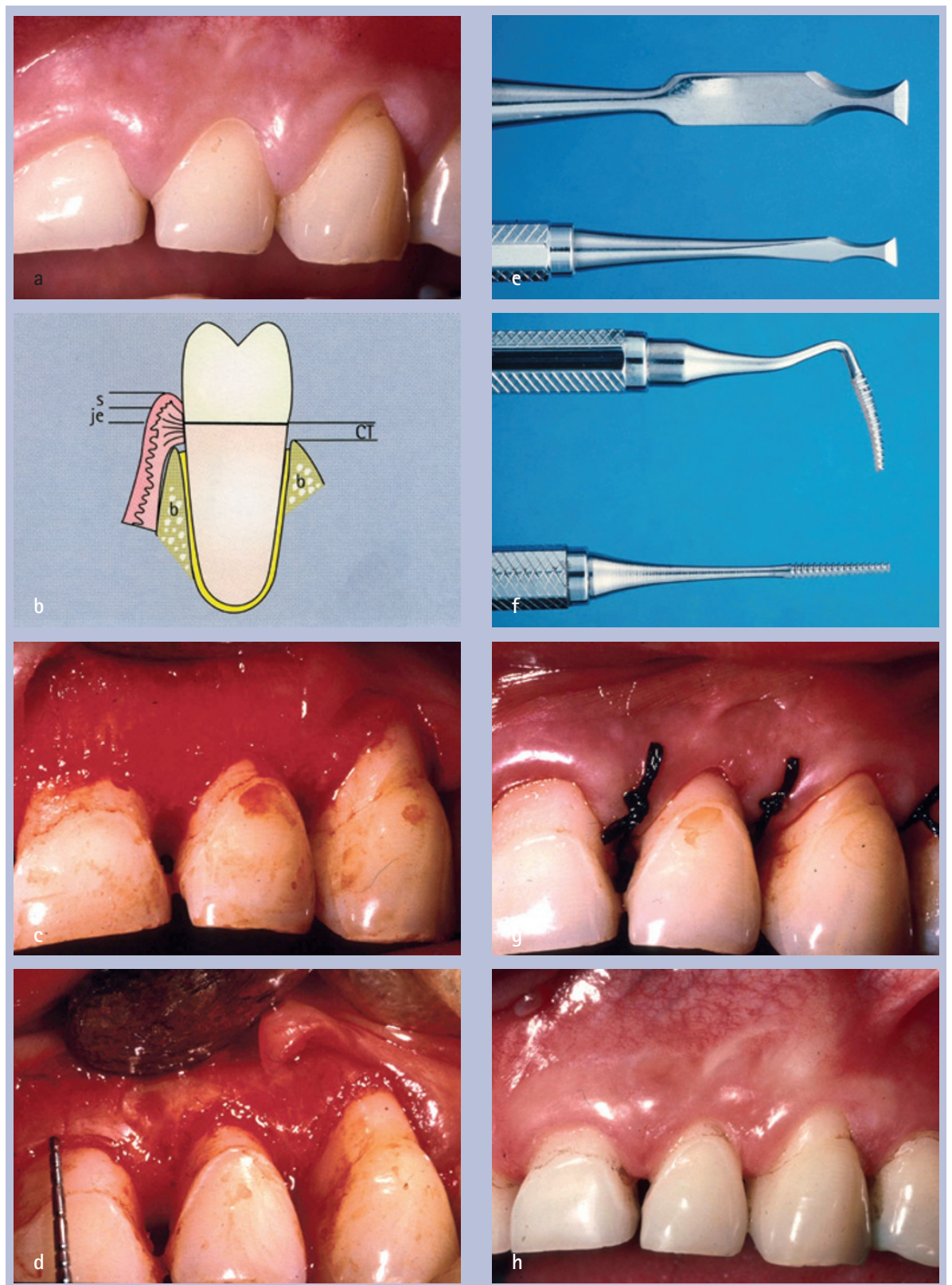

Fig. 3 a) Short clinical crowns due to severe attrition/erosion and a wide zone of attached gingiva. b) Diagram of the dentogingival junction showing approximate dimensions of the gingival attachment apparatus; s, gingival sulcus $(0.5-1 \mathrm{~mm})$; je, junctional epithelium (1-1.5 mm); CT, connective tissue attachment $(2 \mathrm{~mm})$. c) An inverse bevel incision is used to resect some of the gingival tissue and the flap reflected to expose the bone margin. d) Removal of the bone crest to re-establish biological width. e) Periodontal hand chisels.

f) Interdental bone file. g) Flap sutured showing increase in crown height. h) Healed result showing increase in clinical crown height

modified to:

- Increase the clinical crown height to give adequate retention for crowns

- Expose subgingival restoration margins/ secondary caries/fractures.

This procedure is illustrated in Figure 3. Severe attrition and erosion often lead to a situation where full-coverage restoration of the teeth is necessary, but the available clinical crown height does not provide sufficient retention following preparation. In many individuals there is compensatory over eruption of the teeth. This also results essential in planning such procedures in order to confirm the length of the roots of the teeth undergoing treatment and to exclude root resorption or fractures preoperatively.

Limited widths of keratinised gingiva should be preserved by using a crevicular incision. Where there is no pocketing at the normal dentogingival junction, any increase in crown height will have to be achieved by apical positioning of the gingival margin following adequate bone removal.

These are very important decisions and should not be left until the surgical appointment. It is strongly recommended that study casts are evaluated to determine how much tooth reduction is proposed during the restorative treatment and how much increase in clinical crown height will be required to achieve this and to provide a retentive preparation. Close cooperation is needed between operators if the surgery and restorations are to be carried out by different individuals.

Following elevation of the flap the distance between the bone margin and the cement-enamel junction can be assessed. In patients who have not lost any periodontal attachment this is about $2 \mathrm{~mm}$ and this exposed root surface has healthy collagen fibres inserted into cementum. With reference to the amount of extra clinical crown height required, alveolar bone has to be removed to a level which will allow a width of approximately 3-4 $\mathrm{mm}$ between the bone crest and the planned level of the gingival margin. It is very important to minimise trauma to the bone and root surface. Thick bone should be thinned using rotating burs and plenty of saline coolant. The thinned bone adjacent to the root surface can be cleaved off using sharp hand chisels such as the Ochsenbein or TGO (Fig. 3e). On anterior teeth bone height should be preserved as much as possible interdentally to support aesthetic papillae. Where interdental bone has to be removed, purpose-designed files are very useful (Fig. 3f).

The flap is then sutured to cover the alveolar bone. When the flap has been apically positioned a periodontal pack is advisable to maintain the flap position during early healing. It should be remembered that crown lengthening the palatal aspects of the maxillary teeth must rely entirely on resection of soft tissue and bone as required, as apical positioning is not possible.

\section{Ridge augmentation}

Pontic areas can be treated by grafting with various synthetic materials or using soft tissue grafts, bone grafts and guided bone regeneration. These principles are illustrated in Figure 4. 


\section{REPLACEMENT OF MISSING TEETH USING DENTAL IMPLANTS}

Osseointegrated implants are a useful addition in the treatment planning and management of patients who have lost teeth or require extraction of teeth because of periodontitis.

There are a number of important areas to consider:

- Biological comparison of teeth and implants

- Inflammatory conditions around teeth and implants

- Management of advanced periodontitis patients with implants.

\section{Biological comparison of teeth and implants}

There are a number of fundamental factors to consider when comparing the biological and physical aspects of teeth and implants, which are summarised in Table 1.

The gingival cuff around an implant abutment may be very well adapted but it lacks the fibrous connective tissue architecture of the normal dentogingival junction. The tooth is superbly adapted to differing functional demands because of the periodontal ligament. Excessive occlusal forces applied to the crown of a natural tooth will result in widening of the periodontal ligament and an increased mobility. Under these circumstances the tooth is better adapted to cope with the increased forces. By contrast, the osseointegrated implant is rigid within the bone, exhibiting no functional mobility. For example, the nature of the osseointegration does not allow orthodontictype movement of an implant and they have therefore been used successfully as orthodontic anchorage for tooth movement. Moreover, osseointegrated implants should not be used for tooth replacement in the growing child/adolescent as this will result in relative submergence of the implant unit in comparison with the continued eruption of the adjacent natural teeth.

Excessive forces on an implant could result in either material fracture (of the superstructure, retaining screws, abutment screws or the implant itself) or loss of bone contact to the implant surface. The latter may be evident as total loss of integration, with loosening of the implant or loss of marginal bone. The latter type of bone loss is less common than bone loss due to peri-implantitis, which is dealt with in the next section.

\section{Inflammatory conditions around teeth and implants}

Inflammation around the soft tissue of implants, the peri-implant mucosa, without loss of bone is called peri-implant mucositis
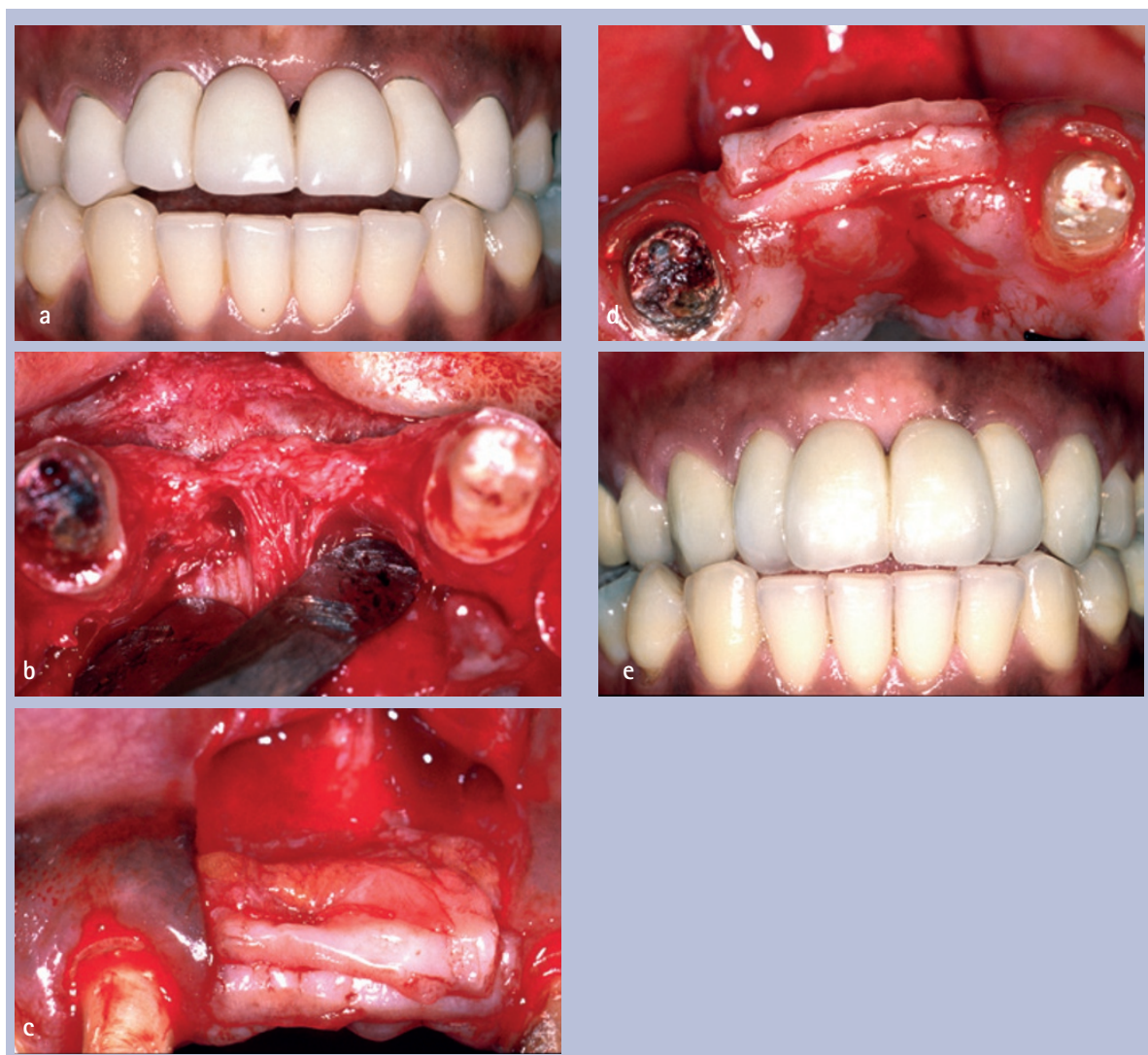

Fig. 4 a) Patient with an anterior maxillary bridge that requires replacement. The edentulous ridge is thin and there is a space between the central incisor pontics where there is a lack of soft tissue. b) The pontic area after flaps have been raised to show the thin bone ridge. c) A double-layered connective tissue graft has been placed to build out the soft tissue profile. d) The thickness of the grafts shown from an occlusal view. e) The patient following provision of a new bridge to show the enhanced soft tissue contour and improvement in aesthetics

Table 1 Healthy teeth versus healthy osseointegrated implants

\begin{tabular}{|c|c|c|}
\hline & Health teeth & Healthy implants \\
\hline Gingival sulcus & Shallow in health (1-3 mm) & $\begin{array}{l}\text { Shallow but dependent upon soft } \\
\text { tissue thickness, abutment length } \\
\text { and position of restoration margin }\end{array}$ \\
\hline Junctional epithelium & On enamel & On surface of implant or abutment \\
\hline Gingival fibres & $\begin{array}{l}\text { Complex array inserted into } \\
\text { cementum coronal to bone crest }\end{array}$ & Parallel fibres with no insertion \\
\hline Crest of bone & $\begin{array}{l}1-2 \mathrm{~mm} \text { apical to the } \\
\text { cement-enamel junction }\end{array}$ & $\begin{array}{l}\text { Variable according to design; for } \\
\text { example, at top of implant or first } \\
\text { thread of implant, at transition } \\
\text { of smooth abutment and rougher } \\
\text { implant surface }\end{array}$ \\
\hline $\begin{array}{l}\text { Connective tissue } \\
\text { attachment }\end{array}$ & $\begin{array}{l}\text { Well-organised collagen fibres } \\
\text { inserted as Sharpey's fibres into } \\
\text { alveolar bone and cementum }\end{array}$ & $\begin{array}{l}\text { Osseointegration - bone growing } \\
\text { in intimate contact with implant } \\
\text { surface layer (titanium oxide, bone } \\
\text { proteoglycan, collagen) }\end{array}$ \\
\hline Physical characteristics & $\begin{array}{l}\text { Physiological mobility due to } \\
\text { viscoelastic properties of the } \\
\text { periodontal ligament }\end{array}$ & $\begin{array}{l}\text { Rigid connection to bone - immobile } \\
\text { as with ankylosis }\end{array}$ \\
\hline Adaptive characteristics & $\begin{array}{l}\text { Width of ligament can alter to allow } \\
\text { more mobility with increased forces }\end{array}$ & $\begin{array}{l}\text { No adaptive capacity to allow } \\
\text { mobility. Orthodontic movement } \\
\text { impossible }\end{array}$ \\
\hline
\end{tabular}

(Fig. 5). It is the equivalent of gingivitis around teeth and is associated with redness and bleeding on probing. It is caused by accumulation of plaque and is managed in the same way as gingivitis. It is important to prevent and control because it may be a precursor to the inflammatory lesion that is associated with bone loss, peri-implantitis. This is the equivalent of periodontitis and is diagnosed when there are inflammatory 


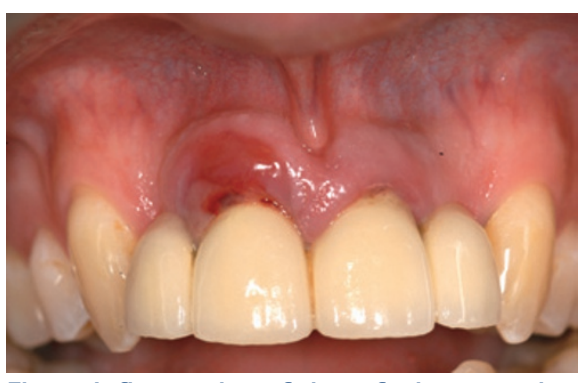

Fig. 5 Inflammation of the soft tissues at the implant restoration at the upper right central incisor. There is no bone loss and a diagnosis of peri-implant mucositis is made

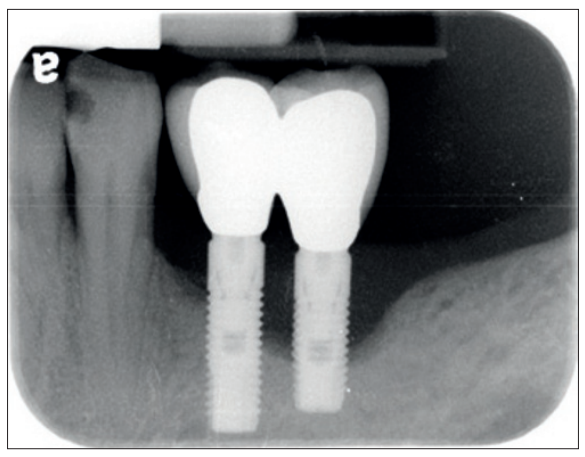

Fig. 6 Radiograph of two implants where there has been extensive loss of bone. This confirms a diagnosis of peri-implantitis, where clinically there are deep pockets and bleeding/ exudate

changes of the peri-implant soft tissue and radiographic evidence of bone loss (Fig. 6). Bleeding on probing and probing depths greater than $4 \mathrm{~mm}$ are present.

The presence of periodontitis affecting remaining teeth or an individual's prior susceptibility to periodontitis may have an important bearing on susceptibility to periimplantitis. Dentate individuals, particularly those with periodontal pockets, will harbour a complex microflora, which is dependent upon an anaerobic environment and nutrient supply from the host tissues. It is likely that bacteria implicated in periodontitis, such as Porphyromonas gingivalis, are also major pathogens in destructive inflammatory lesions around implants. There is therefore a real possibility of colonisation or infection of the implant surfaces from pre-existing periodontopathic bacteria.

The destruction of the supporting tissues of teeth and implants have considerable similarities but there are important differences due to the nature of the supporting tissues. This is particularly noticeable with the patterns of tissue destruction observed. Periimplantitis most commonly affects the entire circumference of the implant, resulting in a 'trough' of bone loss filled with inflammatory tissue extending to the bone surface. By contrast, periodontitis-affected teeth have more irregular loss of supporting tissues, often confined to proximal surfaces and resulting

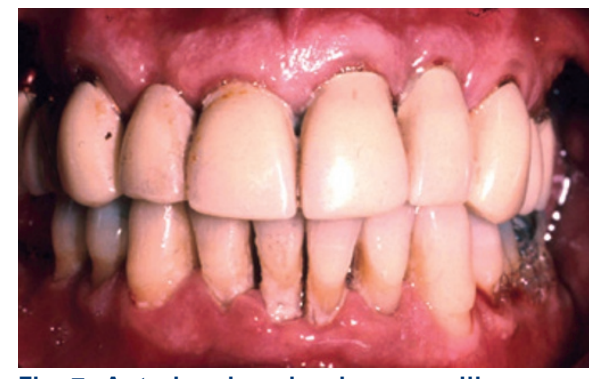

Fig. 7 Anterior view showing a maxillary bridge extending from second premolar to second premolar. The gingival tissues are very inflamed and there are abundant plaque deposits. There is advanced periodontitis affecting the upper and lower dentition

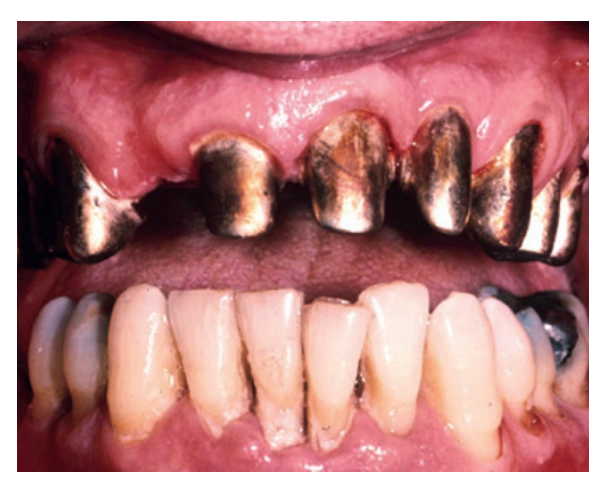

Fig. 8 The uncemented bridge superstructure has been removed to reveal the gold copings on the abutment teeth

in complex infrabony defects. In addition and for the most part, the periodontal tissues are capable of 'walling off' the inflammatory lesion from the alveolar bone and periodontal ligament with a zone of fibrous tissue, a feature which is not seen in peri-implantitis. It would seem probable that destructive inflammatory lesions affecting both teeth and implants have stages in which the disease process is more rapid (burst phenomenon) followed by periods of relative quiescence.

The diagnosis and recognition of periimplantitis would appear to be increasingly common, and may be difficult to manage using existing periodontal non-surgical and surgical techniques. It is important, therefore, that all patients treated with implants are managed in such a way as to avoid this complication. This should be considered at all stages in the treatment process:

- Initial diagnosis and treatment planning

- Preparatory treatment, including extractions, non-surgical and surgical periodontal treatment

- The osseointegration period and any transitional stage

- Maintenance of the implant supported prosthesis.

\section{Management of the patient with advanced periodontitis}

All patients with periodontal disease should be managed appropriately. Only teeth that have severe disease and that are considered to have a hopeless prognosis should be considered for implant replacement. Unfortunately, there has been a trend for more radical management where teeth with treatable periodontitis are removed in favour of implant replacements. This is not only a serious ethical issue but in many cases the patients may suffer from the same complications of inflammatory destruction of support of the implants, which is more difficult to manage than periodontitis.

Treatment of the teeth with periodontitis allows an assessment of patient compliance with oral hygiene procedures, their acceptance of treatment interventions and the tissue response and prognosis. This should better inform the clinician and patient about treatment strategies for replacement of teeth that are missing or need to be replaced. These advantages are lost in cases where the teeth are 'exchanged for implants' in rapid treatment protocols. The following case illustrates periodontal and implant management of a patient with severe periodontitis where the remaining upper teeth have a hopeless prognosis but most of the lower dentition is retained and conventional fixed bridge replacements used.

\section{CASE REPORT}

A case is presented of a patient with advanced periodontitis who had been treated 15 years previously with extensive bridgework in the upper jaw (Fig. 7). Treatment had involved provision of a telescopic reconstruction that was originally cemented to gold copings on the prepared abutment teeth (Fig. 8). The superstructure, however, had been uncemented for several years.

Examination of the individual abutment teeth revealed excessive mobility (over grade 3), very deep pockets and radiographic loss of bone to the root apices and beyond. A limited number of teeth were amenable to treatment. The 13, 11, 21, 23, in the upper jaw had a poor long-term prognosis but could be treated and utilised as interim abutments for a provisional bridge. The 45, 44, 43, $33,34,35$ in the lower jaw were treatable and could be retained as a shortened dental arch and abutments for a tooth-supported anterior bridge. The remaining teeth required extraction. The patient requested restoration of the dentition with fixed bridges without an intervening period of removable dentures. This could be achieved using a transitional approach with provisional tooth-supported bridges in the upper jaw or extraction of all remaining upper teeth with immediate implant placement and immediately loaded implant-supported bridges. The former approach was preferred due to the location of 
adequate bone volume for implant placement and suitable interim tooth abutments. A provisional treatment plan to commence periodontal stabilisation and carry out more detailed evaluation is shown in Table 2.

The definitive treatment plan (Table 3 ) is complex and time consuming with a number of advantages and disadvantages, which should be appreciated (Table 4).

\section{Table 2 Provisional treatment plan}

1. Plaque control instruction and supra- and subgingival scaling

2. Radiographic evaluation of the upper jaw: a. Long-cone parallel radiographs of $13,11,21$, 23 to confirm suitability as interim abutment teeth

b. CT scan of maxillary bone to assess possibility of implant placement

3. Evaluation of diagnostic casts and trial set-up

4. Case discussion and definitive treatment planning. Plan agreed with patient to provide implant-supported upper bridge (using 13,

$11,21,23$ as interim abutments) and toothsupported lower bridge

\section{Table 3 Definitive treatment plan}

1. Construction of an immediate replacement bridge for the lower jaw supported by $45,44,43$, $33,34,35$

2. Modification of the existing upper bridgework to allow extraction of all teeth with the exception $13,11,21,23$

3. Periodontal surgical treatment of remaining teeth to achieve:

a. Pocket elimination and clinical crown lengthening

b. Establishment of a healthy dentogingival junction

4. Modifications of upper bridge and re-preparation of abutment teeth $(13,11,21,23)$ to improve retention (Fig. 9)

5. First stage of implant surgery to place implants at sites 14, 12, 22, 24 using a stent as guide for optimum placement (Fig. 10). The upper bridge pontics to be modified to allow recementation immediately after the surgery allowing for postoperative swelling

6. Postoperative care and continuing periodontal maintenance

7. After a suitable healing (osseointegration) period uncover implants placed at 14, 12, 22, 24 (Fig. 11) and refit provisional bridge

8. Modify bridge to allow connection to implants at 14, 12, 22, 24 (Fig. 12) and extract remaining upper teeth. Provisional bridge now supported by four implants

9. Allow healing of soft tissue to cover extraction sockets (6-8 weeks) and place additional implants at 13 and 23 (unpredicted treatment required [new provisional bridge] Figure 13)

10. Allow integration period before exposing implants at 13 and 23 (Fig. 14) and connecting healing abutments

11. Final restorative phase to provide implantsupported bridge based upon six implants at $14,13,12,22,23,24$ (Figs 15-18) and definitive tooth-supported bridge
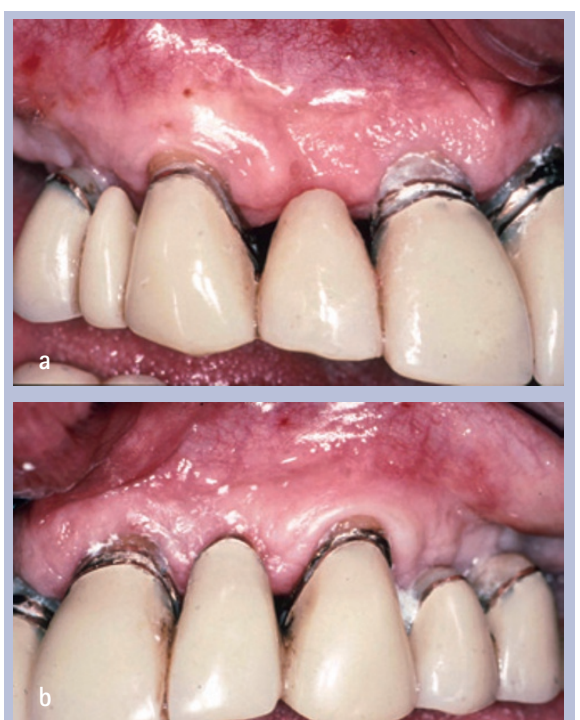

Fig. 9 Right (a) and left (b) views of the modified upper bridgework and the healed periodontal tissues following surgery. The teeth have reduced periodontal support but the tissues are healthy

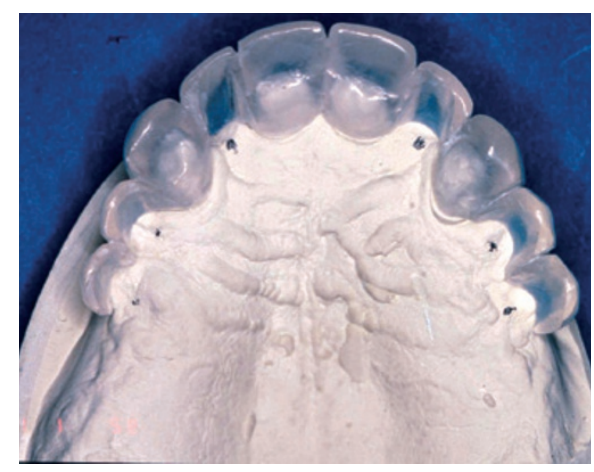

Fig. 10 Surgical stent made to fit abutment teeth copings and providing labial faces of missing teeth to guide implant placement. Ideally, implants would have been placed additionally at the second premolar sites (marks on model), but there was insufficient quantity of bone to allow this

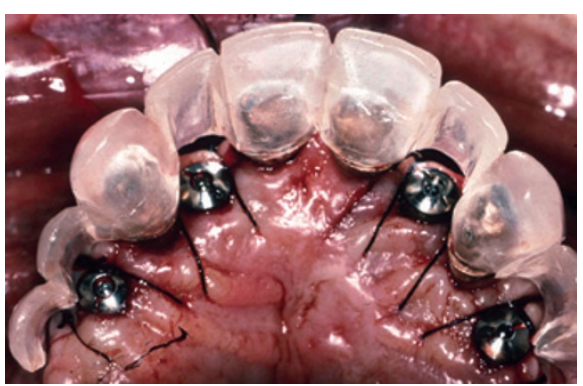

Fig. 11 Implants at 14, 12, 22, 24 (shown in mirror view) have been surgically exposed (stage 2 surgery) and healing abutments attached to them. The surgical stent has been placed on the abutment teeth to demonstrate the favourable position of the implants. The healing abutments are titanium cylinders that pass through the mucosa and protrude about $1 \mathrm{~mm}$. The provisional bridge pontics usually need to be modified to allow recementation immediately after the surgery, allowing for postoperative swelling
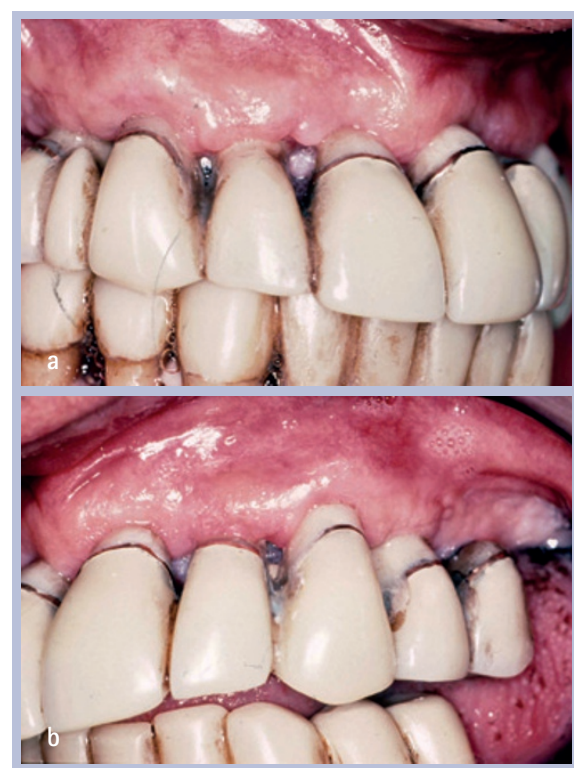

Fig. 12 The bridge has been connected to the implants at $14,12,22,24$ via abutments and screw connection. At this stage the remaining upper teeth are removed and the provisional bridge modified accordingly

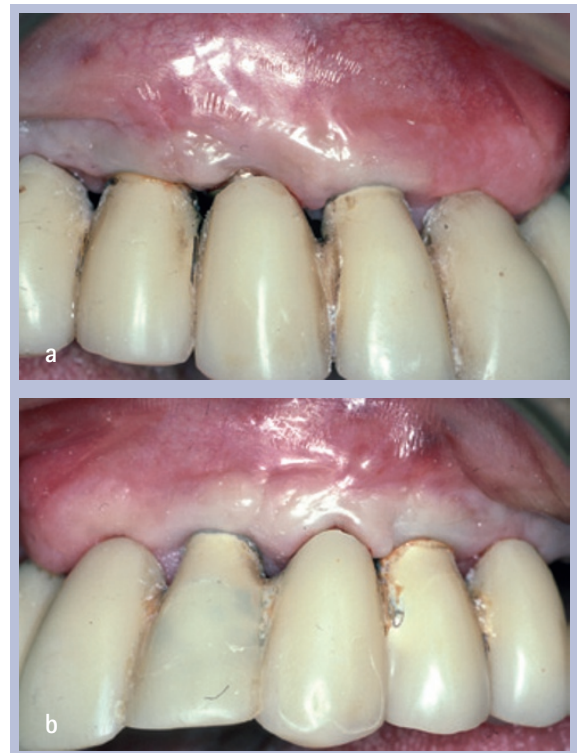

Fig. 13 New provisional bridge connected to implants. The old provisional bridge had been modified to such an extent that breakage was inevitable and a new one had to be made. This was not predicted in the treatment plan

\section{CONCLUSION}

Periodontal management of patients is a key to the long-term success of all treatment plans, but becomes particularly important in patients who are susceptible to periodontitis and those undergoing complex treatment plans. Techniques borrowed from periodontics can also facilitate management of some commonly encountered problems in other dental specialities. 

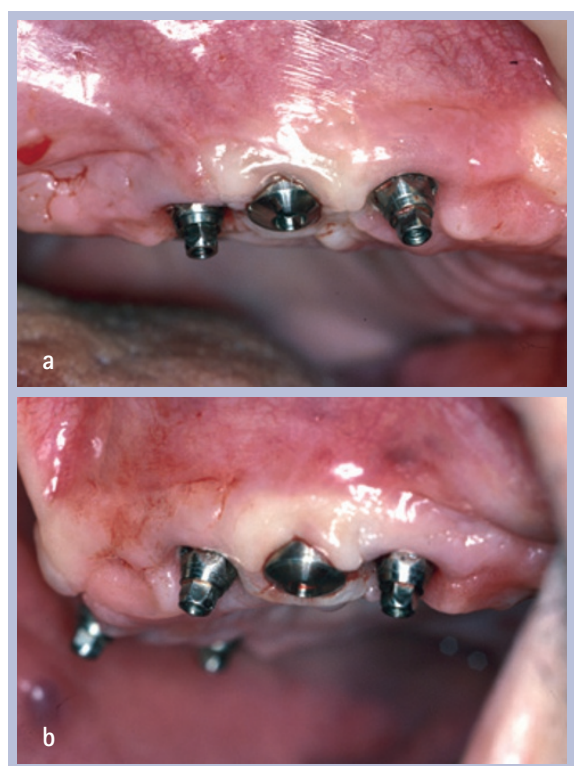

Fig. 14 The implants at 13 and 23 have been exposed and healing abutments have been connected. The abutments on the implants at 14, 12, 22, 24 are to provide screw retention to the provisional bridge

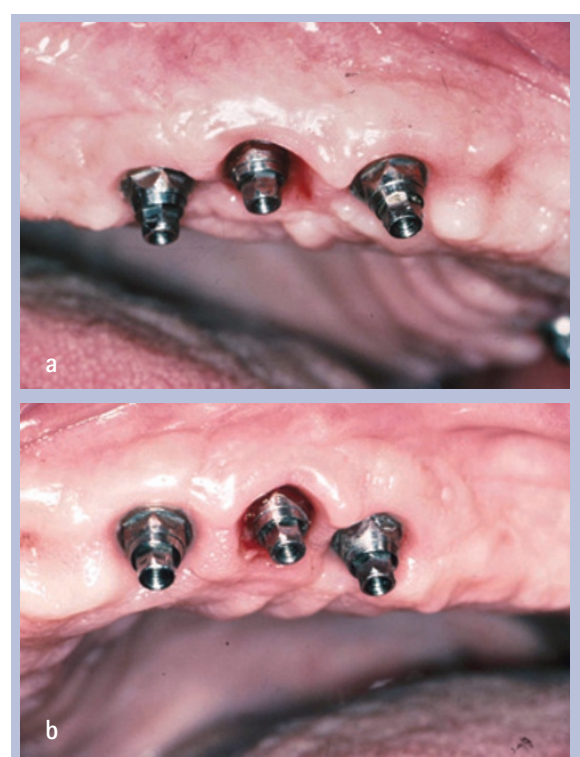

Fig. 15 Abutments have been connected to all the implants and the impressions for the final restoration can be made

\section{FURTHER READING}

\section{Systematic reviews/reviews}

Berglundh T, Zitzmann N U, Donati M. Are periimplantitis lesions different from periodontitis lesions? J Clin Periodontol 2011; 38: 188-202.

Esposito M, Grusovin M G, Worthington H V. Interventions for replacing missing teeth: treatment of peri-implantitis. Cochrane Database Syst Rev 2012 Issue 1: CD004970.

Heitz-Mayfield L J A. Peri-implant diseases: diagnosis and risk indicators. J Clin Periodontol 2008; 35: 292-304.

Kotsovilis S, Karoussis I K, Trianti M, Fourmousis I. Therapy of peri-implantitis: a systematic review. J Clin Periodonto/ 2008; 35: 621-629.

Lang N P, Bosshardt D D, Lulic M. Do mucositis lesions

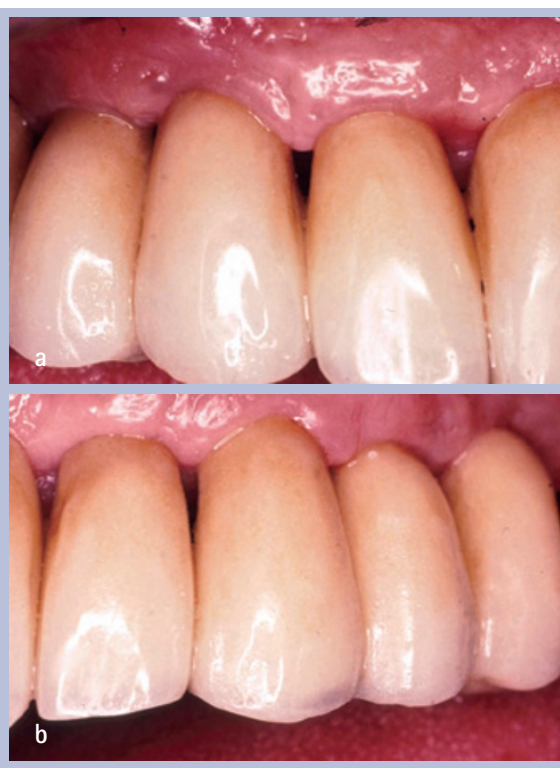

Fig. 16 Fitting of the finished definitive bridgework. The crown margins are just subgingival and the embrasures are shaped to allow proper access for plaque control procedures

around implants differ from gingivitis lesions around teeth? J Clin Periodontol 2011; 38: 182-187.

Mombelli A, Décaillet F, The characteristics of biofilms in peri-implant disease. J Clin Periodontol 2011; 38: 203-213.

Renvert S, Polyzois I, Claffey N. How do implant surface characteristics influence peri-implant disease? J Clin Periodonto/ 2011; 38: 214-222.

Renvert S, Roos-Jansåker A M, Claffey N. Nonsurgical treatment of peri-implant mucositis and periimplantitis: a literature review. J Clin Periodonto/ 2008 35: 305-315.

Zitzmann N U, Berglundh T. Definition and prevalence of peri-implant diseases. J Clin Periodonto/ 2008; 35: 286-291.

\section{Background/historical literature}

Garguilo A W, Wentz F M, Orban B. Dimensions of the dento-gingival junction in humans. J Periodonto/ 1961; 32: $261-267$.

Lundgren D. Prosthetic reconstruction of dentitions seriously compromised by periodontal disease. J Clin Periodontol 1991; 18: 390-395.

Maynard J G, Wilson R D. Physiological dimensions of the periodontium significant to the restorative dentist $J$ Periodontol 1979; 50: 170-174.

Melsen B, Agerbaek N, Markenstam G. Intrusion of incisors in adult patients with marginal bone loss. Am J Orthod 1989; 96: 232-241.

Nyman S, Lindhe J. A longitudinal study of combined periodontal and prosthetic treatment of patients with advanced periodontal disease. J Periodonto/ 1979; 50: 163-169.

Polson A M. The relative importance of plaque and occlusion in periodontal disease. J Clin Periodontol 1986 13: 923-927.

Williams S, Melsen B, Agerbaek N, Asboe V. The orthodontic treatment of malocclusion in patients with previous periodontal disease. Br J Orthod 1982; 9: 178-184.

Wise M D. Stability of gingival crest after surgery and before anterior crown placement. J Prosthet Dent 1985; 53: 20-23.

The authors would like to acknowledge Adam Hasan and Tim Newton as specialist contributors to A clinical guide to periodontology, 3rd edition.

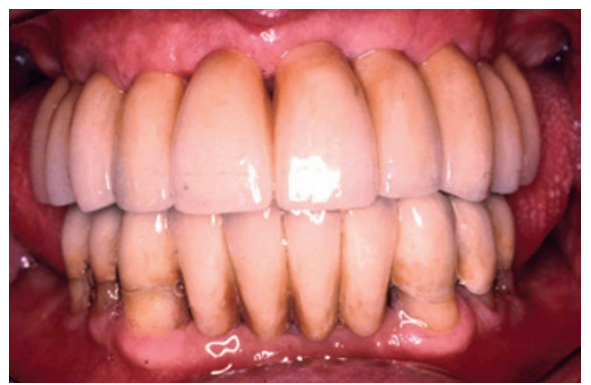

Fig. 17 Anterior intraoral view of the completed upper implantsupported and lower tooth-supported bridges

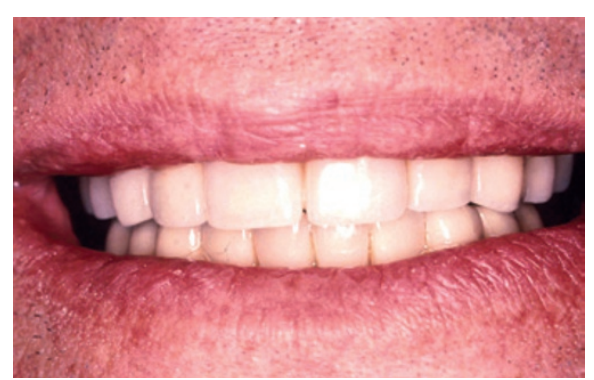

Fig. 18 Extraoral view of the completed restoration

Table 4 Advantages and disadvantages of the treatment plan

Advantages

- The patient is able to wear a fixed bridge throughout treatment, including the immediate post-surgical phase(s)

- It is particularly advantageous in those cases where guided bone regeneration techniques with implanted membranes have been used because the overlying tissues are not subject to the forces and trauma of a mucosal-borne denture

- Development and control of the occlusion is obtained at an early stage in treatment

\section{Disadvantages}

- The planning is difficult and time consuming. It may be difficult for the patient to appreciate the large number of stages and the time involved

- The treatment is costly and complex, requiring a great deal of interplay between restorative and surgical disciplines

- It may be necessary to construct several bridges to satisfactorily complete the treatment; for example:

- Immediate insertion bridge with wire reinforcement and adaptation with resin to accommodate tooth extraction

Provisional bridge with metal substructure and resin superstructure following periodonta treatment. This bridge is constructed to last over the complete treatment period until the final bridge is inserted. This could be over a timescale of over one year

- An additional provisional bridge may be required to allow connection of the 'first wave' of implants

- The final bridge

- The additional cost of the numerous bridges and extra surgical procedures is obvious. The additional time required removing and replacing provisional bridges on many occasions is sometimes overlooked 